\title{
Theory and Linguistic Practice in the 21st Century —Epistemologically Regarded as the Post-Classical Linguistic Investigations
}

\author{
Oleksandr Oguy \\ National University of Chernivtsi, Chernivtsi, Ukraine
}

\begin{abstract}
The paper is dedicated to the analysis of linguistic methodology. Being based on analytical-synthetical method, the author defines (due to Mario Bunge) three main types of linguistic approaches. Classical one is orientated on gathering data, un-/neo classical (structural) approach is directed to defining the mechanism of their organization, and post-classical (or postmodernist) approach promotes investigating complex systems in their self-development. The main qualities of it are synergy, emergency, symmetry, self-organization, etc. expressed by qualitative and quantitative parameters of order. The author illustrates some of them. On this basis the complex-integrated investigation of the language and a new ELT-teaching are proposed as a main achievement. The combining of these three approaches allows a new holistic theory for linguistics in perspective. It can be comprehended as a deductive-inductive systemic set of proposals based on empirical knowledge touching upon a given thing (semantics) and predicating its possible development. This theory is to be also empirically provable and organized as a deductive-hypothetical logical system for receiving logical predictions and reconstruction of linguistic events. It is high time to combine the neurolinguistic and psycholinguistic, functional and structural, and synergetic approaches with quantitative and qualitative characteristics of the object (phonetics, lexicology, semasiology, grammar, theory of translation).
\end{abstract}

Keywords: linguistic methodology, linguistic approaches: classical, neo classical, post-classical

\section{Introduction}

Once Ferdinand de Saussure (1949) said: "La tâche de la linguistique sera:... de se délimiter et de se définir elle meme” (p. 20), i.e., the main task of it is to find limits (boundaries) and define itself under other kinds of science, because he clearly understood the common way of scientific development. The epistemology (the science of science) well known in Western-European and rather rarely used in Eastern-European culture is dedicated to finding several common features and special differences in every kinds of science and culture too (philosophy, arts, geography, biology, physics, etc.). As we know Karl Popper and Mario Bunge (philosophers), G. Haken (biologist), Illya Prigozhin (the Nobel Prize laureate in physics: unstable thermodynamics), V. Paszhenko (geographer), Werner Welte, Raimund Koehler, Boris Parshin, V. Kybrik et al. (linguists) achieved 
some perspective interdisciplinary results in its comprehension. These results can enrich every object under study. That is why it is necessary to define the main approaches to the scientific investigation of language and to find the fields of their usage. These approaches following each other historically in every kind of science are known in epistemological investigations such as: classical, un-/neo classical (structural) and post-classical (or postmodernist) ones. They can be differed by the means of their purpose and methods for achieving it.

\section{Types of Linguistic Investigation}

\section{The Classical Investigation}

It is well known to everybody the primary purpose of every scientific investigation is the development of knowledge. It can take place if the explorer answers the questions "Who? What? Where? When? Why? In what direction?” touching upon some object. Such asking and answering questions are only the "children age” of every science when "its concentration on the search for singular data, classification, relevant variables and isolated hypothesis, establishing relationships among these variables, as well as accounting for those data” (Bunge, 1967, p. 380) is characteristic. The attention of the scientist is concentrated on simple concrete objects (Bunge, 1983, p. 35), i.e., traditional for this atomic approach are logical methods of abstraction, analysis, synthesis, induction, analogy, measure, and observation. Based on these attempts a lot of empiric generalizations and new information appeared serving for accumulation of our knowledge in patterns of various variables. Epistemologically observed the result received is not of best quality. Bunge (1967) remarked ironically: "A heap of uncoordinated hypotheses may be likened to a huge mass of protoplasm without a nervous system” (p. 382), i.e., the achieved results are unsatisfied because of uncoordination of its components. As to the language, its typical patterns of this kind are: hermeneutic, philological, and linguistic ones (Chebanov \& Martynenko, 1988, p. 112) demonstrating the use of language in texts (ph.), its units (l.), and their hidden content (h.).

\section{The Non-classical Investigation}

However, this epoch of "the data-gathering-and-packaging view of science" (Bunge, 1967, p. 383) for the linguistics was overcome in the second non-classical period. It is founded on the complex investigation of the object's system as a complicated set by means of deductive reflexing. Its main problems are: "What is it combined with? What is its principle? What is it for?” As the result of using traditional methods (such as the systematization or taxonomination, abstraction, analysis, hypothetic-deductive method, etc.) some non-classical approaches appeared. They are due to Paschenko (2000, pp. 66-70): constructive, systematic, ecological, scientologic, global, cybernetic, sociologic, substantional, semiotic, informative, semantic, modeling, and structural ones. As to the language the Swiss linguist Ferdinand de Saussure (1857-1913) having used the structural approach systematized the ideas of it. He found logic consequences between the linguistic objects and could explain in this way a lot of empiric data. His bilateral dichotomic system (langue-parole, synchrony-diachrony, syntagmatics-paradigmatics, significant-signifée, etc.) was able to explain some systemic factors (but not the direction of their development!). The most of modern linguistic investigations have been carried out in this direction without pretending to create a new theory after Saussure (besides the pragmatic attempt of Morris and later Searle et al. based on the ideas of Dewes). In the post-classical period of development of all sciences, the linguistics has reached only the usual classical investigation of the system (due to Saussure) without having taken into consideration its synergetyc properties. The paradigms of the classical and 
non-classical linguistic researches seem to be exhausted nowadays under new conditions. The received system improved our knowledge while producing new propositions as hypotheses, improved their testability and could serve for building up a new deductive theory.

\section{The Post-non-classical Investigation}

Neoclassical (post-non-classical) or postmodernist conceptions unite classical and non-classical approaches on three levels of cognition: philosophic-methodological, common scientific, and concrete scientific in their completeness. They propose the most perspective scientifically holistic synergetic paradigms (Welte, 1993, p. 36), promoting our investigating complex systems in their self-development. The main problem of this approach is the following one: "How is this system self-developing? In what direction? What are the perspectives of it?” For such an approach, some principles combined with spatial-temporal organization of systematically organized phenomena under study (ergodicity, self-organization, self-development, synergy, symmetry, emergency, etc.) being the property not of a desperate object, but of its complexes (Bunge, 1983, p. 37) are of great significance. Due to last systematic investigations every system (e.g., biological, physical, technical, geographical, language) consists of specific configurations (modes) changing under unstable outer parameters due to these fundamental principles: emergency, synergy, self-organization, etc.. (Haken, 1980, pp. 34-35; Paschenko, 2000, p. 180). Synergy (Greek synergeia, Lat. synergetus) is common cooperative action of different factors necessary for self-organization of object (Paschenko, 2000, p. 208). Emergency is essential property of a structured object to be more than the sum of its structural components thanks to a new-appeared synergetic quality (Paschenko, 2000, p. 186). Self-organization can be regarded as totality of processes serving for creating, re-creating, preserving, and improving the organization of a complicated dynamic system (based on self-regulation) (Paschenko, 2000, p. 180). These properties appear and grow up in the holistic object due to the effect of the holistic organization.

Parameters of order. As compared with physics, biology, chemistry, economy, etc., the use of the post-modernist approach is dedicated to the looking for the directions of language self-development, and its wholessness, dynamics, emergency, synergy, etc. leaves much to be desired. Raimund Koehler, the only linguist engaged in synergetic research, has found several "parameters of order" (quantitative data for defining the state of phenomenon) for the whole language, but not for its properties (like polysemy with its vague boundaries). That is why several parameters of order demonstrating the synergy of linguistic phenomena have been found for German. The coefficient of polysemy (Cp) (found on the materials of Duden and Wahrig) appeared to be hierarchically organized for different parts of speech in modern German (verb-adjective-noun-adverb: 2,37:1,27:1,24:1,13). Such organization is being observed in many Indo-European, Finno-Ugric, and Turkish languages. The increasing of the average coefficient, e.g., for adjectives from 1,27 till 2,19 (in Middle-High-German period) promotes the depolysemizational process when some words loose their meanings. Only $15 \%$ of words being polysemantically try to cover $85 \%$ of the text. The average frequency of German frequent adjectives found based on Ortmann appeared to be 530 word usages (WU—for a monosemantic word). Though the absolute frequency of polysemantic words is 1,623 WU, but the average frequency of one meaning of a polysemantic word providing the communication is near to the mentioned above-571 WU. So called multi-semed words with only one wide (often vague) meaning had an increased frequency—till 900 WU (Oguy, 
2000). These parameters demonstrate, e.g., the super-systematic interstructural relationships of language between frequency and meanings.

As far as we see during the lasting post-classical period of development of all sciences (physics, geography, biology, etc.), the linguistics has reached only the classical level of investigation of the system (due to Saussure) without having taken into consideration its synergetic and holistic properties. That is why according to the materials of the conference "Linguistics at the end of the 20th century: Results and perspectives" (Moscow, 1996) it is high time to combine the neurolinguistic and psycholinguistic, functional and structural, and synergetic approaches with quantitative and qualitative characteristics of the object. Due to the combined results of the linguistics II (of neurolinguistic kind), the meaning of a word is not a simple synaptic-molecular product of a separate definite part of brain, but a phenomenon of extremely high-coordinated and complicated functioning of all its systems. It can appear as a real linguistic object (with its paradigmatic, syntagmatic, and epidigmatic relations) only as a holistic function of the whole brain. That is why it is necessary to combine the neurolinguistic and psycholinguistic, functional and structural, and synergetic approaches with quantitative and qualitative characteristics of the object (phonetics, lexicology, semasiology, grammar, theory of translation). It means a new holistic approach as a construction of multilateral analytical linguistic characteristics in a synthetic description is to be introduced. That is why it is high time to found a postclassical unique deductive theory of linguistics.

Complex-integrated investigation of the language. But we know in the post-classical period of development of all sciences, the linguistics has reached only the usual classical investigation of the system (due to Saussure) without having taken into consideration its synergetic properties. It seems quite possible to be overcome with a new approach. Such an approach can be based upon the fixation of linguistic units, definition of their differential signs and quantitative variables, description of the contentual potential of these units, taking into consideration the peculiarities of the systemic relations and communicative situation and if possible the neurophysiological specifics of their functioning. Such a complex-integrated investigation of the language in this aspect can have as a result a new holistic revaluation of functioning of the linguistic phenomena (semantics) and that is why it can serve for creation of a new synergetic conception of the language with hermeneutic interpretation of its cognitive properties.

As to the linguistics, it is not able (despite many systematical, but departed conceptions) to observe the word and meaning because the holistic systematization and explanation of some empiric facts are absent despite many to pity unsatisfied attempts. If the principle of deductive input of our knowledge is taken into consideration, the systematization must be fulfilled on three levels: Mega, Macro, and Micro. In this case the language is Mega-level for a semantic phenomena or meaning, and the semantics is the Macro-level. The integration of the data received on the level (with inclusion of the data of Mega and Micro levels) can systematically serve for final purpose "the building of progressively truer partial reconstructions of reality" (Bunge, 1967, p. 29).

New ELT-teaching. It is high time to leave the classic (though reliable) pattern of ELT-teaching and create a new synergetic or holistic one, promoting the language communication on new level of competence. This synergy and emergency appear on different levels (linguistic, methodological, and psychological) of ELT process (Filippova, 2003, pp. 149-150). They can be linguistically achieved by integrated introduction of literality, history, and theory of EFL for focusing interdisciplinar functional-grammatical, lexical, cognitive, and cultural aspects in constructing the contents of a speech act. Methodologically the synergy can occur when different 
methods of ELT are used for effective instruction of communicative competent speaker. Psychologically the synergetism is based upon holistic involving different instruction strategies for effective individual and collective activities (as multiple intelligences, learning styles).

A new holistic theory for linguistics in perspective. On this basis there is still room for hope a new holistic theory for linguistics can appear:

A set of scientific hypotheses is a scientific theory if only it refers to a given factual subject matter and every member of the set is either an initial assumption or a logical consequence of one or more initial assumptions. (Bunge, 1967, p. 381; Bunge, 1987, p. 145)

Such a theory can be comprehended as a deductive-inductive systemic set of propositions based on empirical knowledge touching upon a given thing and predicating its possible development (Bunge, 1987, pp. 145, 380). In order to be scientifical, this theory is to be also empirically provable and organized as a deductive-hypothetical logical system for receiving logical predictions and reconstruction of linguistic events (cf. Bunge, 1987, p. 145). It is high time to combine the neurolinguistic and psycholinguistic, functional and structural, and synergetic approaches with quantitative and qualitative characteristics of the object (phonetics, lexicology, semasiology, grammar, theory of translation). In such a way, the scientific (linguistic) theory can be observed "as an activity or as a finished even though not final product of that activity” (Bunge, 1967, p. 380) useful for practice (incl. ELT or historical studies, etc.).

\section{References}

Bunge, M. (1967). Scientific research I: The search for system (p. 536). Berlin, Heidelberg, \& New York: Springer.

Bunge, M. (1983). Epistemologie: Aktuelle Fragen der Wissenschaftslehre (p. 217). Mannheim, Wien, \& Zürich: Bibl.Institut.

Bunge, M. (1987). Kausalität, Geschichte und Probleme (Causality, history and problems) (p. 217). Tübingen: Mohr.

Chebanov, S. W., \& Martynenko, G. J. (1990). Osnownyje tipy predstawlenija o prirode jasyka (Main types of ideas about the nature of language) (pp. 112-132). Tartu: Uc. zap. Tartus. uniwers. 911.

De Saussure, F. (1949). Cours de Linguistique generale (Course of common linguistics) (p. 420). Paris: Payot.

Filippova, N. (2003). Sinergetika u suchasniy metodytsi (Synergetics in metodics of nowadays). International scientific conference „Prykladna linguistyka u 21 stolitti: Linguodydaktyczni ta kulturolohichni aspekty” (pp. 149-150). Lviv: Stavropigion.

Haken, G. (1980). Sinergetika (Synergetics) (p. 404). Moskow: Mir.

Moser, F. (1989). Bewusstsein in Raum und Zeit. Grundlagen einer holistischen Weltauffassung auf wissenschaftlicher Basis (Consciousnessin space and time. Foundations of the holistic outlook on the scientific basis) (p. 250). Graz: Leykam.

Oguy, O. D. (2000). Systemno-kvantytatyvni aspekty... (Systematic-quantitative aspects of polysemy in German (Synchrony, Diachrony and Panchrony)) (p. 450). (Theses for achieving the degree of Full Professor in specialty 10.02.04-Germanic languages). Kyiv.

Paschenko, V. M. (2000). Metodolohiya... (Methodology of natural-geographical knowledge) (p. 318). Kyiv.

Welte, W. (1993). Englische Semantik: Ein Lehr- und Arbeitsbuch mit umfassender Bibliographie (English semantics: A teaching and working book with expanded bibliography) (p. 405). Frankfurt a.M., Berlin, \& New York: P. Lang. 